\title{
Application of green-extraction technique to evaluate of antioxidative capacity of wild population of fireweed (Epilobium angustifolium)
}

\author{
ANNA NOWAK ${ }^{1}$, ADAM KLIMOWICZ ${ }^{1}$, WIKTORIA DUCHNIK ${ }^{1}$, ŁUKASZ KUCHARSKIㄴ, \\ KATARZYNA FLORKOWSKA ${ }^{1}$, ANNA MUZYKIEWICZ ${ }^{1 *}$, DARIA WIRA ${ }^{1}$, JOANNA ZIELONKA- \\ BRZEZICKA $^{1}$, ANITA SIEDŁOWSKA ${ }^{2}$, KINGA NADARZEWSKA ${ }^{2}$
}

Chair and Department of Cosmetic and Pharmaceutical Chemistry

Pomeranian Medical University

Al. Powstańców Wielkopolskich 72

70-111 Szczecin, Poland

${ }^{2}$ Student Research Group

Chair and Department of Cosmetic and Pharmaceutical Chemistry

Pomeranian Medical University

Szczecin

*corresponding author: phone: +48 501483 469; e-mail: anna.muzykiewicz@pum.edu.pl

\section{Summary}

Introduction: Fireweed (Epilobium angustifolium (L.) Holub) is a common weed growing on meadows, roadside and agricultural wasteland, creating vast, rapidly spreading fields.

Objective: The aim of the study was to evaluate the antioxidant activity of extracts from fresh fireweed, harvested at three ripening stages.

Methods: Analysis of antioxidative activity was carried out by DPPH, ABTS and FRAP methods. Total polyphenol and total flavonoid content were also determined. Plant material was extracted using ultrasound-assisted green extraction technique with methanol, ethanol and isopropanol at different concentrations and water.

Results: The highest antioxidant activity evaluated by DPPH, ABTS and FRAP was found for the extracts prepared in $70 \%$ ethanol. The highest content of total polyphenols were observed in extracts in $70 \%$ ethanol, whereas the highest content of flavonoids extracts in undiluted methanol.

Conclusion: Epilobium angustifolium harvested at fruit ripening stage seems to be a valuable source of antioxidants. 


\section{Keywords: Epilobium angustifolium, antioxidant activity, total polyphenol content, total flavonoid content, green-extraction technique}

Słowa kluczowe: Epilobium angustifolium, aktywność antyoksydacyjna, ogólna zawartość polifenoli, ogólna zawartość flawonoidów, zielona metoda ekstrakcji

\section{INTRODUCTION}

Fireweed (Epilobium angustifolium (L.) Holub) belongs to the family of Onagraceae and occurs mainly in North America, Asia and Europe. This plant belongs to the genus Epilobium, consisting of more than 200 species, with 26 different varieties of this plant in Europe [1-3]. Its major natural habitat are coniferous forests, although it can often be found on porches, gravel lands, wastelands, roadsides, meadows and pastures, as well as in highly insolated areas [4]. This plant is an undesirable weed, especially in fields and meadows, due to its high competitiveness to other plant species to access nutrients and water. It has been recommended in traditional medicine for a long time as a popular raw material with analgesic, antibacterial and anti-inflammatory activity. Moreover, it has been also used as an adjuvant in the prevention and alleviation of symptoms of prostatic hyperplasia. Due to the sweet taste, this plant is popular in Russia as a infusion from fermented leaves and used as an adjuvant for gastric ulcerations and inflammations [5-6]. Many authors have also observed antioxidant properties of E. angustifolium. An increasing consumption of plants with antioxidant capacity seems to be important. The formation of an excessive amount of reactive oxygen species (ROS) can cause an oxidation of proteins, lipids and DNA and can lead to cell damage. As a consequence, susceptibility to many so-called civilization diseases such as, for example, cardiovascular and neoplastic diseases, as well as diabetes, osteoporosis and neurodegenerative diseases has been observed [7-9].

One of modern extraction techniques used to obtain plant material, inter alia active ingredients with antioxidant properties, is the application of ultrasound-assisted extraction, which could be classified as so-called green extraction method. Recently, this technique has been increasingly used due to its high efficiency. Extraction of plant material occurs in a short time, along with reduced consumption of solvents, coupled with lower environmental pollution [10]. It is an affordable, simple and efficient method, as compared to traditional extraction techniques.
Ultrasounds exert a mechanical effect to allow an increase of solvent penetration into the sample matrix and to increase the contact area between solid and liquid phase. As a result, the substances contained in the plant material quickly diffuse to the extraction solvent [11]. The aim of the study was to evaluate the antioxidant activity and the total polyphenol and flavonoid content of extracts of fireweed, harvested from the natural state, in three plant ripening stages. The ultrasound-assisted extraction was performed in 15, 30 and 60 minutes with use of four solvents (water, methanol, ethanol and isopropanol in different proportions) to obtain the extracts.

\section{MATERIAL AND METHODS}

Reagents. 2,2-diphenyl-1-picrylhydrazyl (DPPH), 6-hydroxy-2,5,7,8-tetramethylchroman-2-carboxylic acid (trolox), 2,2-azino-bis (ethylbenzothiazolin-6-sulfonoic acid (ABTS), 2,4,6-tripyridyl-S-triazine (TPTZ) and rutin were purchased from Sigma Aldrich (USA); Folin-Ciocalteu reagent from Merck (Germany); 99.5\% acetic acid, sodium acetate anhydrous, potassium persulfate, potassium acetate, aluminum chloride, $36 \%$ hydrochloric acid, as well as ethanol, methanol and isopropanol (all of analytical grade) were obtained from Chempur, Piekary Śląskie (Poland).

Plant material. The plant material consisted of fresh fireweed harvested in 2017 from a natural site, which was an agricultural wasteland located near a

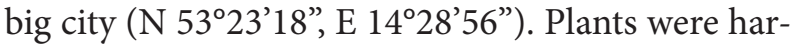
vested in three developmental stages: the first - intensive growing (second week of May), the second - massive blooming (second week of July) and the third - fruit ripening stage (fourth week of August). The harvested material was subjected to extraction immediately. The plant material was identified by Anna Nowak, who graduated from Agriculture University, Szczecin, Poland. Her PhD thesis concerned plant physiology and her research was closely relater to this field of interest.

Ultrasound-assisted extraction. The vegetable raw material was extracted using the following solvents: 
aqueous ethanol $(40 \% v / v), 70 \% v / v)$ and undiluted), aqueous methanol ( $40 \% v / v), 70 \% v / v$ and undiluted), aqueous isopropanol $(40 \% v / v), 70 \% v / v$ and undiluted) as well as water. Extraction was performed using an ultrasound bath for 15, 30 or 60 minutes. The obtained extracts were evaluated for their antioxidant activity and for total polyphenol and flavonoid content using spectrophotometric methods.

DPPH radical scavenging activity. The scavenging activity of DPPH stable free radicals was measured as described previously $[12,13]$. Shortly, a sample of $0.15 \mathrm{~cm}^{3}$ of the plant extract was mixed with $2.85 \mathrm{~cm}^{3}$ of $0.3 \mathrm{mM}$ DPPH radical solution dissolved in $96 \% v / v$ ethanol. Measurement of antioxidant activity was performed after $10 \mathrm{~min}$. of incubation in dark at a room temperature. Absorbance at $517 \mathrm{~nm}$ was measured. The results are presented as radical scavenging activity (RSA) [\%].

ABTS radical scavenging activity. The procedure applied to evaluate ABTS radical scavenging activity was described previously [14]. Shortly, $7 \mathrm{mM}$ solution of ABTS (2,2-azine-bis (3-ethylbenzthiazoline6-sulfonic acid) in a $2.45 \mathrm{mM}$ aqueous solution of potassium persulfate was used to prepare the stock solution. After dissolving the components, solution was incubated for 24 hours in dark at a room temperature, then diluted with $50 \% v / v$ methanol to obtain an absorbance of $1.000 \pm 0.005$. The abovementioned solution was added to the test extract in a ratio of 1:100 by vol. The absorbance of the samples was measured at $734 \mathrm{~nm}$. As previously, the results were expressed as trolox equivalent antioxidant capacity - TEAC.

FRAP assay. The ability to reduce ions $\mathrm{Fe}^{3+}$ to $\mathrm{Fe}^{2+}$ (Ferric ion Reducing Antioxidant Power - FRAP) was determined as previously described [15]. In this method, the working solution consisted of $0.3 \mathrm{M}$ acetate buffer ( $\mathrm{pH}$ 3.6) mixed with $0.01 \mathrm{M} \mathrm{2,4,6-tri-}$ pyridyl-S-triazine (TPTZ) in $0.04 \mathrm{M} \mathrm{HCl}$ and $0.02 \mathrm{M}$ ferric chloride (10:1:1 by vol.). One volume of plant extract was mixed with 29 parts of this solution. The absorbance was measured at a wavelength of $593 \mathrm{~nm}$ and the results were expressed as TEAC.

Total polyphenols content (TPC). Total polyphenol content was determined with Folin-Ciocalteau method as described previously [12]. Shortly, to $0.15 \mathrm{~cm}^{3}$ of the extract $0.15 \mathrm{~cm}^{3}$ of tenfold diluted Folin-Ciocalteu reagent, $1.35 \mathrm{~cm}^{3}$ of $0.01 \mathrm{M}$ sodium carbonate solution and $1.35 \mathrm{~cm}^{3}$ water was added and mixed. After 15 min of incubation in dark at a room temperature, the absorbance was measured at $765 \mathrm{~nm}$. Gallic acid (GA) was applied as a standard and results were expressed as gallic acid equivalents
(GAE) in mg gallic acid/g of raw material.

Total flavonoid content. The total flavonoid content in the test samples was determined using the colorimetric method described by Berreira et al. [16]. Shortly, to $2.5 \mathrm{~cm}^{3}$ of plant extract, $1.25 \mathrm{~cm}^{3}$ distilled water and $0.075 \mathrm{~cm}^{3}$ of $5 \%$ sodium nitrite solution were added. After $5 \mathrm{~min}, 0.15 \mathrm{~cm}^{3}$ of $10 \%$ aluminum chloride and $0.5 \mathrm{~cm}^{3}$ of $1 \mathrm{M}$ sodium hydroxide aqueous solution were added followed by $1.35 \mathrm{~cm}^{3}$ water. As a reference substance, rutin (RU) was used. The results were expressed in $\mathrm{mg} \mathrm{RU} / \mathrm{g}$ of raw material. Spectrophotometric measurements were taken at $510 \mathrm{~nm}$.

Statistical analysis. The statistical analysis of the results was carried out with the Statistica 12 program package (StatSoft) using a one-way analysis of ANOVA variance, with the significance level $\mathrm{p}<0.05$. Inter-group differences were determined by Tukey's test $(n=3)$. The Pearson correlation coefficient between the results obtained with individual methods of antioxidative capacity assessment and total polyphenols and flavonoids content were also calculated. To evaluate the differences between individual ripening stages, the Wilcoxon test was used.

Ethical approval: The conducted research is not related to either human or animal use.

\section{RESULTS AND DISCUSSION}

When assessing the antioxidant activity of the plant raw material, the solvent used, the extraction time, as well as the method of evaluation of antioxidant potential seems to be important $[13,14]$. In the present study, the extraction of the raw material was carried out by ultrasound assisted extraction, classified as the so-called green extraction method. In recent years, more and more attention has been paid to the use of ecological methods to obtain plant extracts. Such techniques are aimed primarily to less solvent consumption, and thus less interference in the natural environment. The ultrasound extraction technique has been previously used by many researchers. Li et al. and Goula et al. isolated carotenoids from carrot root (Dacutus carota) and pomegranate fruit (Punica granatum) $[17,18]$. Chemat et al. found that isolation of active substances using an ultrasonic bath was a key technology to achieve sustainable "green" chemistry due to, among others, a short time of extraction and low energy consumption [19].

In our study, commonly used techniques, such as $\mathrm{DPPH}, \mathrm{ABTS}$, FRAP were applied to evaluate 
antioxidant activity. DPPH (2,2-diphenyl-1-picrylhydrazyl) forms a stable radical to be reduced in the presence of antioxidants and leads to decolourization of violet to form a pale yellow solution. The method is used to determine antioxidant potential of either individual compounds as well as plant extracts [20-22]. Another technique frequently used to assess the antioxidant activity is based on the application of ABTS (2,2-azine-bis (ethylbenzthiazoline-6-sulphonic acid) radical. This method allows to determine the antioxidant activity of both hydrophobic and hydrophilic antioxidants [22]. In the present study, antioxidant activities measured by DPPH method showed a similar tendency to results obtained by ABTS, however, the activity values expressed as TEAC using ABTS method were significantly higher as compared to DPPH method. Similar relationships between methods were also observed in our previous study on antioxidative activity of green tea [14]. In contrary, Wojdyło et al. observed results dissimilar to our for E. hirsutum, using trolox as a standard in both methods, because the values obtained by DPPH method were significantly higher, as compared to the results obtained with ABTS technique [21].

In present study, the antioxidant activity measured with use of DPPH method ranged from $13.42 \% \pm 0.86$ for $15 \mathrm{~min}$ aqueous extracts in fruit ripening stage to $96.77 \% \pm 4.77$ for samples prepared in 70\% ethanol in $15 \mathrm{~min}$ (also fruit ripening stage). With this method, high values were also found for extracts prepared in $96 \%$ (30 $\mathrm{min}$ ) and 70\% ethanol (60 $\mathrm{min})$ as well as in 70\% methanol (15 min): $94.12 \% \pm 0.87 ; 94.03 \% \pm 0.09$ and $93.80 \% \pm 0.73$, respectively, for material harvested in the fruit-setting state. When ABTS method was applied, the TEAC values ranged from $0.61 \pm 0.01$ for extracts in undiluted isopropanol (60 $\mathrm{min}$; fruit ripening stage) to $59.64 \pm 0.57$ for extracts in $70 \%$ ethanol (extraction time $60 \mathrm{~min}$ ) harvested during intensive growing stage) (tab. 1).

E. augustifolium is known as a plant with high antioxidant activity [1, 5, 22-25]. Tóth et al. used the DPPH method to assess the antioxidant activity of aqueous extracts and those of $80 \%$ acetone in various species of willow (E. parviflorum, E. roseum, E. tetragonum, E. montanum and E. angustifolium). They observed antioxidant activity expressed as $\mathrm{EC}_{50}$ of $7.96 \pm 0.24 \mu \mathrm{M}$, while for ascorbic acid $\mathrm{EC}_{50}$ it was $14.29 \pm 0.43 \mu \mathrm{M}$ [25]. Wojdyło et al. found antioxidant activity of E. hirsutum herb extracts in $80 \%$ methyl alcohol. They analyzed 32 species of medicinal plants, belonging to 21 botanical families, to be found as wild plants in Poland. They observed antioxidant activity of fireweed to be one of the highest, as compared to other plants. The antioxidant activity of E. hirsutum, measured by the DPPH method, was $2021 \mu \mathrm{M}$ trolox/100 g dry matter. In contrary, activity of garden angelica (Archangelica officinalis) was only $7.34 \pm 1.14 \mu \mathrm{M}$ trolox/100 g dry matter. Similarly, the highest value of $69.5 \mu \mathrm{M}$ trolox/100 g dry matter was also found for E. hirsutum with ABTS method. Based on the results of the study, the authors came into conclusion that the majority of analyzed plant species, naturally occurring in Poland, have lower activity, as compared to fireweed. In most cases, it was lower than $500 \mu \mathrm{M}$ trolox/100 g dry matter [21]. Also Stef et al. compared antioxidant potential of E. hirsutum with several other plants. They prepared extracts of eleven medicinal plants in $50 \%$ ethanol. The DPPH radical scavenging activity was $21.87 \%$ for E. montanum and it was slightly lower than that of purple coneflower (Echinacea purpurea): $23.43 \%$ and herb wormwood (Artemisia absinthium): 22.93\% [26].

The antioxidant effect of plant materials can also be assessed by FRAP method, the principle of which is based on the measurement of TPTZ (iron2,4,6-tripyridyl-S-thiazine complex) reduction by antioxidant contained for instance in plant [27]. In our study it was shown that extracts from fireweed have the ability to reduce $\mathrm{Fe}^{3+}$ ions, the highest TEAC values of $97.50 \pm 0.72$ and $102.52 \pm 1.33$ were found for extracts from the plant harvested in the intensive growing stage, prepared in $70 \%$ ethanol in 30 and $60 \mathrm{~min}$, respectively (tab. 2). Wojdyło et al. also demonstrated that the antioxidative activity of E. hirsutum extracts in $80 \%$ methanol determined by the FRAP method, was $275 \mu \mathrm{M}$ trolox/100 g [21]. Moreover, Stef et al. used FRAP technique and found the ability of E. montanum extracts in $50 \%$ ethanol to reduce iron ions of $4.28 \mathrm{Fe}^{2+} \mathrm{mM} / 1$ [26].

The active compounds of plants, such as polyphenols or flavonoids, could influence their biological activity. Such compounds are one of the most popular groups of nutrients, classified as phytochemicals, to have a protective effect against many diseases [28]. Fireweed belongs to plants rich in polyphenols and flavonoids $[1,3,21,25,27,29]$. This observation has been also confirmed in our studies. The total polyphenol content ranged from $0.38 \pm 0.09 \mathrm{GAE}$ for water extracts, extracted in 30 minutes (intensive growing stage) to $22.99 \pm 0.12$ GAE for extracts prepared in $70 \%$ ethanol, extraction time 30 min (fruit ripening stage) (tab. 4). The highest total flavonoid content of $7.03 \pm 0.20 \mathrm{mg}$ $\mathrm{RU} / \mathrm{g}$ of raw material was observed for fruit ripening 
Table 1.

Mean ( \pm standard deviation) antioxidant activity of fresh E. angustifolium herb extracts (in \%RSA) evaluated with DPPH method.

\begin{tabular}{|c|c|c|c|c|}
\hline \multicolumn{5}{|c|}{$\begin{array}{c}\text { DPPH } \\
{[\% \text { RSA }]}\end{array}$} \\
\hline \multirow{2}{*}{ Solvent } & \multirow{2}{*}{$\begin{array}{c}\text { Alcohol } \\
\text { concentration } \\
{[v / v]}\end{array}$} & \multicolumn{3}{|c|}{ Extraction time } \\
\hline & & $15^{\prime}$ & $30^{\prime}$ & $60^{\prime}$ \\
\hline \multicolumn{5}{|c|}{ Intensive growing } \\
\hline \multirow{3}{*}{ Methanol } & $99.8 \%$ & $87.94 \pm 0.95 \mathrm{ab}$ & $85.69 \pm 1.66 \mathrm{~b}$ & $88.04 \pm 0.39 b$ \\
\hline & $70 \%$ & $84.64 \pm 2.09 \mathrm{~b}$ & $78.32 \pm 2.50 \mathrm{c}$ & $80.05 \pm 1.55 \mathrm{c}$ \\
\hline & $40 \%$ & $72.24 \pm 1.74 b$ & $76.93 \pm 3.48 b$ & $82.06 \pm 0.57 b$ \\
\hline \multirow{3}{*}{ Ethanol } & $96 \%$ & $87.29 \pm 1.33 \mathrm{a}$ & $86.48 \pm 0.83 b$ & $88.52 \pm 0.48 \mathrm{c}$ \\
\hline & $70 \%$ & $75.94 \pm 4.28 \mathrm{a}$ & $83.15 \pm 0.47 b$ & $86.92 \pm 0.41 \mathrm{~b}$ \\
\hline & $40 \%$ & $78.42 \pm 1.86 \mathrm{~b}$ & $77.23 \pm 1.33 \mathrm{c}$ & $83.69 \pm 1.57 b$ \\
\hline \multirow{3}{*}{ Isopropanol } & $99.5 \%$ & $79.78 \pm 1.75 b$ & $73.36 \pm 2.50 \mathrm{~b}$ & $74.99 \pm 1.77 \mathrm{~b}$ \\
\hline & $70 \%$ & $81.92 \pm 0.58 \mathrm{c}$ & $84.00 \pm 3.02 b$ & $77.95 \pm 3.96 b$ \\
\hline & $40 \%$ & $81.72 \pm 0.16 \mathrm{~b}$ & $85.25 \pm 0.39 b$ & $79.17 \pm 1.94 b$ \\
\hline \multicolumn{2}{|l|}{ Water } & $27.79 \pm 3.47 b$ & $27.42 \pm 1.15 b$ & $39.72 \pm 0.56 c$ \\
\hline \multicolumn{5}{|c|}{ Massive blooming } \\
\hline \multirow{3}{*}{ Methanol } & $99.8 \%$ & $86.32 \pm 1.82 \mathrm{~b}$ & $84.89 \pm 3.06 \mathrm{~b}$ & $89.05 \pm 2.54 \mathrm{~b}$ \\
\hline & $70 \%$ & $78.21 \pm 2.61 \mathrm{c}$ & $85.43 \pm 1.24 \mathrm{~b}$ & $85.81 \pm 1.62 \mathrm{~b}$ \\
\hline & $40 \%$ & $73.27 \pm 0.81 \mathrm{~b}$ & $79.80 \pm 2.14 \mathrm{~b}$ & $86.65 \pm 3.94 \mathrm{ab}$ \\
\hline \multirow{3}{*}{ Ethanol } & $96 \%$ & $86.49 \pm 3.57 \mathrm{a}$ & $87.10 \pm 1.30 \mathrm{~b}$ & $90.36 \pm 0.75 b$ \\
\hline & $70 \%$ & $78.90 \pm 2.71 \mathrm{a}$ & $87.32 \pm 2.96 \mathrm{~b}$ & $89.59 \pm 3.07 \mathrm{ab}$ \\
\hline & $40 \%$ & $77.42 \pm 1.88 \mathrm{~b}$ & $82.23 \pm 2.02 \mathrm{~b}$ & $84.28 \pm 2.34 \mathrm{~b}$ \\
\hline \multirow{3}{*}{ Isopropanol } & $99.5 \%$ & $54.73 \pm 2.20 \mathrm{c}$ & $33.01 \pm 3.06 \mathrm{~b}$ & $86.33 \pm 1.80 \mathrm{a}$ \\
\hline & $70 \%$ & $89.74 \pm 0.45 b$ & $89.91 \pm 0.58 \mathrm{a}$ & $88.38 \pm 2.22 \mathrm{a}$ \\
\hline & $40 \%$ & $82.22 \pm 0.99 b$ & $87.38 \pm 0.53 b$ & $87.99 \pm 3.00 \mathrm{a}$ \\
\hline \multicolumn{2}{|l|}{ Water } & $35.54 \pm 1.79 \mathrm{a}$ & $39.07 \pm 2.64 \mathrm{a}$ & $81.36 \pm 0.63 b$ \\
\hline \multicolumn{5}{|c|}{ fruit ripening } \\
\hline \multirow{3}{*}{ Methanol } & $99.8 \%$ & $92.34 \pm 3.57 \mathrm{a}$ & $91.48 \pm 1.66 \mathrm{a}$ & $92,62 \pm 1,10 \mathrm{a}$ \\
\hline & $70 \%$ & $93.80 \pm 0.73 \mathrm{a}$ & $91.26 \pm 0.58 \mathrm{a}$ & $93,92 \pm 0,44 \mathrm{a}$ \\
\hline & $40 \%$ & $92.88 \pm 0.38 \mathrm{a}$ & $90.88 \pm 1.23 \mathrm{a}$ & $90,47 \pm 0,33 \mathrm{a}$ \\
\hline \multirow{3}{*}{ Ethanol } & $96 \%$ & $86.53 \pm 1.18 \mathrm{a}$ & $94.12 \pm 0.87 \mathrm{a}$ & $93,38 \pm 0,24 a$ \\
\hline & $70 \%$ & $96.77 \pm 4.77 \mathrm{a}$ & $93.70 \pm 0.15 \mathrm{a}$ & $94,03 \pm 0,09 a$ \\
\hline & $40 \%$ & $91.71 \pm 0.48 \mathrm{a}$ & $92.40 \pm 0.44 \mathrm{a}$ & $93,42 \pm 0,05 a$ \\
\hline \multirow{3}{*}{ Isopropanol } & $99.5 \%$ & $88.43 \pm 4.15 \mathrm{a}$ & $77.75 \pm 4.43 \mathrm{a}$ & $87,91 \pm 0,20 \mathrm{a}$ \\
\hline & $70 \%$ & $93.35 \pm 2.32 \mathrm{a}$ & $93.16 \pm 1.57 \mathrm{a}$ & $92,62 \pm 1,56 a$ \\
\hline & $40 \%$ & $92.88 \pm 1.90 \mathrm{a}$ & $91.33 \pm 2.52 \mathrm{a}$ & $92,69 \pm 2,55 \mathrm{a}$ \\
\hline \multicolumn{2}{|l|}{ Water } & $13.42 \pm 0.86 c$ & $17.76 \pm 2.40 c$ & $90.91 \pm 0.97 a$ \\
\hline
\end{tabular}

The values marked with different letters differ significantly between the development stages $(p<0.05, \mathrm{n}=3)$ 
Table 2.

Mean ( \pm standard deviation) antioxidant activity of fresh E. angustifolium herb extracts (in TEAC, i.e. mg trolox/g raw material) evaluated with ABTS method.

\begin{tabular}{|c|c|c|c|c|}
\hline \multicolumn{5}{|c|}{$\begin{array}{c}\text { ABTS } \\
{[\text { TEAC, } \mathrm{mg} \text { trolox/g raw material] }}\end{array}$} \\
\hline \multirow{2}{*}{ Solvent } & \multirow{2}{*}{$\begin{array}{c}\text { Alcohol } \\
\text { concentration } \\
{[v / v]}\end{array}$} & \multicolumn{3}{|c|}{ Extraction time } \\
\hline & & $15^{\prime}$ & $30^{\prime}$ & $60^{\prime}$ \\
\hline & & tensive growi & & \\
\hline \multirow{3}{*}{ Methanol } & $99.8 \%$ & $27.26 \pm 0.30 \mathrm{a}$ & $25.71 \pm 1.93 \mathrm{a}$ & $10.30 \pm 1.14 \mathrm{c}$ \\
\hline & $70 \%$ & $34.35 \pm 0.77 \mathrm{~b}$ & $50.00 \pm 1.46 \mathrm{a}$ & $32.57 \pm 2.26 \mathrm{~b}$ \\
\hline & $40 \%$ & $25.40 \pm 0.58 b$ & $41.94 \pm 0.93 b$ & $20.07 \pm 1.08 \mathrm{~b}$ \\
\hline \multirow{3}{*}{ Ethanol } & $96 \%$ & $2.77 \pm 0.47 \mathrm{~b}$ & $24.98 \pm 2.27 \mathrm{a}$ & $34.75 \pm 0.68 \mathrm{a}$ \\
\hline & $70 \%$ & $24.33 \pm 0.54 a$ & $59.06 \pm 0.90 \mathrm{a}$ & $59.64 \pm 0.57 \mathrm{a}$ \\
\hline & $40 \%$ & $31.02 \pm 1.29 \mathrm{~b}$ & $36.63 \pm 1.79 b$ & $57.86 \pm 0.61 \mathrm{a}$ \\
\hline \multirow{3}{*}{ Isopropanol } & $99.5 \%$ & $14.58 \pm 0.71 b$ & $16.23 \pm 0.27 \mathrm{a}$ & $15.36 \pm 0.64 a$ \\
\hline & $70 \%$ & $9.48 \pm 1.83 \mathrm{c}$ & $28.77 \pm 1.18 b$ & $41.34 \pm 1.27 \mathrm{a}$ \\
\hline & $40 \%$ & $10.61 \pm 1.54 \mathrm{c}$ & $23.73 \pm 0.41 b$ & $16.56 \pm 0.26 c$ \\
\hline Water & & $10.47 \pm 0.58 \mathrm{a}$ & $9.90 \pm 0.23 \mathrm{a}$ & $10.26 \pm 0.59 a$ \\
\hline \multicolumn{5}{|c|}{ Massive blooming } \\
\hline \multirow{3}{*}{ Methanol } & $99.8 \%$ & $17.97 \pm 0.59 b$ & $15.00 \pm 1.57 \mathrm{~b}$ & $20.16 \pm 0.19 b$ \\
\hline & $70 \%$ & $20.50 \pm 0.37 c$ & $16.74 \pm 1.21 \mathrm{~b}$ & $21.06 \pm 0.29 c$ \\
\hline & $40 \%$ & $20.92 \pm 0.25 c$ & $21.22 \pm 0.10 c$ & $21.25 \pm 0.01 b$ \\
\hline \multirow{3}{*}{ Ethanol } & $96 \%$ & $7.64 \pm 0.49 a$ & $7.19 \pm 0.05 c$ & $15.22 \pm 0.58 b$ \\
\hline & $70 \%$ & $11.72 \pm 0.41 b$ & $21.05 \pm 0.10 \mathrm{c}$ & $21.14 \pm 0.18 c$ \\
\hline & $40 \%$ & $20.11 \pm 0.23 c$ & $17.30 \pm 0.20 c$ & $20.80 \pm 0.11 b$ \\
\hline \multirow{3}{*}{ Isopropanol } & $99.5 \%$ & $5.31 \pm 0.39 c$ & $1.97 \pm 0.41 \mathrm{c}$ & $7.05 \pm 0.85 b$ \\
\hline & $70 \%$ & $21.15 \pm 0.21 b$ & $21.25 \pm 0.10 c$ & $20.95 \pm 0.42 b$ \\
\hline & $40 \%$ & $17.57 \pm 0.60 \mathrm{~b}$ & $20.86 \pm 0.25 c$ & $21.09 \pm 0.04 \mathrm{~b}$ \\
\hline Water & & $2.08 \pm 0.03 \mathrm{~b}$ & $2.58 \pm 0.09 b$ & $4.39 \pm 0.42 b$ \\
\hline \multicolumn{5}{|c|}{ Fruit ripening } \\
\hline \multirow{3}{*}{ Methanol } & $99.8 \%$ & $17.43 \pm 1.31 \mathrm{~b}$ & $22.79 \pm 1.72 \mathrm{a}$ & $39.15 \pm 0.99 a$ \\
\hline & $70 \%$ & $44.57 \pm 0.57 \mathrm{a}$ & $49.28 \pm 1.49 a$ & $54.66 \pm 1.16 \mathrm{a}$ \\
\hline & $40 \%$ & $56.91 \pm 0.33 \mathrm{a}$ & $53.69 \pm 1.24 \mathrm{a}$ & $53.83 \pm 1.62 \mathrm{a}$ \\
\hline \multirow{3}{*}{ Ethanol } & $96 \%$ & $8.47 \pm 0.89 a$ & $16.97 \pm 1.20 \mathrm{~b}$ & $15.95 \pm 1.00 \mathrm{~b}$ \\
\hline & $70 \%$ & $6.68 \pm 0.80 c$ & $41.49 \pm 1.11 \mathrm{~b}$ & $45.13 \pm 1.48 b$ \\
\hline & $40 \%$ & $46.22 \pm 1.99 a$ & $40.52 \pm 1.39 a$ & $58.74 \pm 1.16 \mathrm{a}$ \\
\hline \multirow{3}{*}{ Isopropanol } & $99.5 \%$ & $39.31 \pm 1.99 a$ & $9.26 \pm 0.23 b$ & $0.61 \pm 0.01 \mathrm{c}$ \\
\hline & $70 \%$ & $30.02 \pm 1.08 \mathrm{a}$ & $42.51 \pm 1.36 \mathrm{a}$ & $38.94 \pm 1.76 \mathrm{a}$ \\
\hline & $40 \%$ & $32.19 \pm 1.19 a$ & $35.32 \pm 0.82 \mathrm{a}$ & $37.30 \pm 1.08 \mathrm{a}$ \\
\hline \multicolumn{2}{|l|}{ Water } & $0.67 \pm 0.07 c$ & $2.33 \pm 0.25 b$ & $10.88 \pm 0.19 a$ \\
\hline
\end{tabular}

The values marked with different letters differ significantly between the development stages $(p<0.05, \mathrm{n}=3)$ 
Table 3.

Mean ( \pm standard deviation) antioxidant activity of fresh E. angustifolium herb extracts (in TEAC, i.e. mg trolox/g raw material) evaluated with FRAP method.

\begin{tabular}{|c|c|c|c|c|}
\hline \multicolumn{5}{|c|}{$\begin{array}{c}\text { FRAP } \\
{[\text { TEAC, mg trolox/g raw material }]}\end{array}$} \\
\hline \multirow{2}{*}{ Solvent } & \multirow{2}{*}{$\begin{array}{c}\text { Alcohol } \\
\text { concentration } \\
{[v / v]}\end{array}$} & \multicolumn{3}{|c|}{ Extraction time } \\
\hline & & $15^{\prime}$ & $30^{\prime}$ & $60^{\prime}$ \\
\hline \multicolumn{5}{|c|}{ Intensive growing } \\
\hline \multirow{3}{*}{ Methanol } & $99.8 \%$ & $17.67 \pm 0.96 a$ & $67.67 \pm 1.04 \mathrm{a}$ & $48.82 \pm 0.39 \mathrm{a}$ \\
\hline & $70 \%$ & $38.90 \pm 0.37 \mathrm{a}$ & $55.73 \pm 0.78 \mathrm{a}$ & $88.53 \pm 0.65 a$ \\
\hline & $40 \%$ & $3.06 \pm 0.04 c$ & $35.77 \pm 0.97 \mathrm{a}$ & $50.12 \pm 0.59 \mathrm{a}$ \\
\hline \multirow{3}{*}{ Ethanol } & $96 \%$ & $18.49 \pm 0.51 \mathrm{a}$ & $16.68 \pm 0.61 b$ & $31.71 \pm 0.91 \mathrm{a}$ \\
\hline & $70 \%$ & $71.76 \pm 1.05 a$ & $97.50 \pm 0.72 \mathrm{a}$ & $102.52 \pm 1.33 \mathrm{a}$ \\
\hline & $40 \%$ & $44.47 \pm 0.58 \mathrm{a}$ & $93.92 \pm 0.24 \mathrm{a}$ & $85.07 \pm 0.85 a$ \\
\hline \multirow{3}{*}{ Isopropanol } & $99.5 \%$ & $6.60 \pm 0.64 b$ & $7.48 \pm 0.39 a$ & $11.97 \pm 0.44 \mathrm{a}$ \\
\hline & $70 \%$ & $32.01 \pm 1.16 \mathrm{a}$ & $40.95 \pm 1.53 \mathrm{a}$ & $53.64 \pm 1.54 \mathrm{a}$ \\
\hline & $40 \%$ & $36.63 \pm 1.81 \mathrm{a}$ & $33.91 \pm 0.89 \mathrm{a}$ & $95.13 \pm 0.33 a$ \\
\hline Water & & $6.43 \pm 0.31 \mathrm{a}$ & $12.85 \pm 1.18 \mathrm{a}$ & $22.62 \pm 0.61 \mathrm{a}$ \\
\hline \multicolumn{5}{|c|}{ Massive blooming } \\
\hline \multirow{3}{*}{ Methanol } & $99.8 \%$ & $19.08 \pm 0.54 \mathrm{a}$ & $12.20 \pm 0.41 \mathrm{c}$ & $17.95 \pm 0.20 \mathrm{c}$ \\
\hline & $70 \%$ & $21.30 \pm 0.56 \mathrm{c}$ & $15.67 \pm 0.26 c$ & $21.25 \pm 0.41 \mathrm{c}$ \\
\hline & $40 \%$ & $21.13 \pm 0.27 b$ & $22.76 \pm 0.57 c$ & $20.48 \pm 0.26 c$ \\
\hline \multirow{3}{*}{ Ethanol } & $96 \%$ & $10.78 \pm 0.43 b$ & $8.64 \pm 0.40 c$ & $16.13 \pm 0.32 c$ \\
\hline & $70 \%$ & $12.55 \pm 0.91 b$ & $24.25 \pm 0.35 c$ & $20.19 \pm 0.30 b$ \\
\hline & $40 \%$ & $19.45 \pm 0.51 \mathrm{c}$ & $19.03 \pm 0.27 \mathrm{c}$ & $20.90 \pm 0.50 c$ \\
\hline \multirow{3}{*}{ Isopropanol } & $99.5 \%$ & $2.13 \pm 0.30 c$ & $0.08 \pm 0.01 b$ & $6.94 \pm 0.29 b$ \\
\hline & $70 \%$ & $22.39 \pm 0.44 c$ & $20.39 \pm 0.34 \mathrm{c}$ & $19.18 \pm 0.25 c$ \\
\hline & $40 \%$ & $15.40 \pm 0.60 c$ & $16.84 \pm 0.21 \mathrm{c}$ & $19.75 \pm 0.42 \mathrm{c}$ \\
\hline Water & & $0.50 \pm 0.06 \mathrm{~b}$ & $0.06 \pm 0.01 \mathrm{~b}$ & n.a. \\
\hline \multicolumn{5}{|c|}{ Fruit ripening } \\
\hline \multirow{3}{*}{ Methanol } & $99.8 \%$ & $17.30 \pm 0.69 a$ & $19.45 \pm 0.64 b$ & $26.82 \pm 0.44 b$ \\
\hline & $70 \%$ & $24.21 \pm 0.59 b$ & $28.12 \pm 0.11 b$ & $27.91 \pm 0.36 b$ \\
\hline & $40 \%$ & $27.90 \pm 0.54 \mathrm{a}$ & $26.19 \pm 0.31 b$ & $42.91 \pm 0.50 \mathrm{~b}$ \\
\hline \multirow{3}{*}{ Ethanol } & $96 \%$ & $8.18 \pm 0.54 c$ & $26.14 \pm 0.66 \mathrm{a}$ & $20.98 \pm 0.83 b$ \\
\hline & $70 \%$ & $6.47 \pm 0.29 c$ & $35.79 \pm 0.61 b$ & $20.98 \pm 0.83 b$ \\
\hline & $40 \%$ & $22.93 \pm 1.08 \mathrm{~b}$ & $33.70 \pm 0.47 \mathrm{~b}$ & $42.78 \pm 0.80 \mathrm{~b}$ \\
\hline \multirow{3}{*}{ Isopropanol } & $99.5 \%$ & $25.01 \pm 0.60 \mathrm{a}$ & $7.05 \pm 0.11 \mathrm{a}$ & $11.43 \pm 0.03 \mathrm{a}$ \\
\hline & $70 \%$ & $28.14 \pm 1.31 b$ & $26.59 \pm 0.65 b$ & $27.52 \pm 0.04 \mathrm{~b}$ \\
\hline & $40 \%$ & $30.12 \pm 1.07 \mathrm{~b}$ & $24.25 \pm 1.00 \mathrm{~b}$ & $26.35 \pm 0.12 b$ \\
\hline \multicolumn{2}{|l|}{ Water } & n.a. & n.a. & $0.95 \pm 0.09 b$ \\
\hline
\end{tabular}

n.a. - no activity. The values marked with different letters differ significantly between the development stages $(p<0.05, \mathrm{n}=3)$ 
Table 4

Mean ( \pm standard deviation) total polyphenols content in fresh E. angustifolium herb extracts (in GAE, i.e. mg GA/g of raw material.

\begin{tabular}{|c|c|c|c|c|}
\hline \multicolumn{5}{|c|}{$\begin{array}{c}\text { FOLIN-CIOCALTEU } \\
{[\mathrm{GAE}, \mathrm{mg} \text { gallic acid/g raw material] }}\end{array}$} \\
\hline \multirow{2}{*}{ Solvent } & \multirow{2}{*}{$\begin{array}{c}\text { Alcohol } \\
\text { concentration } \\
{[v / v]}\end{array}$} & \multicolumn{3}{|c|}{ Extraction time } \\
\hline & & $15^{\prime}$ & $30^{\prime}$ & $60^{\prime}$ \\
\hline \multicolumn{5}{|c|}{ Intensive growing } \\
\hline \multirow{3}{*}{ Mmethanol } & $99.8 \%$ & $5.50 \pm 0.23 b$ & $8.28 \pm 0.23 a$ & $7.63 \pm 0.30 b$ \\
\hline & $70 \%$ & $5.73 \pm 0.33 b$ & $7.26 \pm 0.04 b$ & $7.75 \pm 0.22 \mathrm{a}$ \\
\hline & $40 \%$ & $1.35 \pm 0.19 b$ & $4.98 \pm 0.16 b$ & $5.61 \pm 0.26 \mathrm{~b}$ \\
\hline \multirow{3}{*}{ Ethanol } & $96 \%$ & $6.10 \pm 0.23 a$ & $5.75 \pm 0.33 a$ & $7.17 \pm 0.12 b$ \\
\hline & $70 \%$ & $8.53 \pm 0.15 a$ & $9.08 \pm 017 b$ & $7.97 \pm 0.28 b$ \\
\hline & $40 \%$ & $6.14 \pm 0.13 b$ & $8.11 \pm 0.21 b$ & $7.81 \pm 0.26 b$ \\
\hline \multirow{3}{*}{ Isopropanol } & $99.5 \%$ & $2.75 \pm 0.27 b$ & $3.57 \pm 0.22 \mathrm{a}$ & $4.07 \pm 0.10 \mathrm{~b}$ \\
\hline & $70 \%$ & $5.85 \pm 0.30 \mathrm{~b}$ & $8.25 \pm 0.18 b$ & $10.18 \pm 0.31 \mathrm{a}$ \\
\hline & $40 \%$ & $4.53 \pm 0.27 b$ & $6.97 \pm 0.24 b$ & $10.32 \pm 0.35 a$ \\
\hline \multicolumn{2}{|l|}{ Water } & $0.51 \pm 0.06 \mathrm{a}$ & $0.19 \pm 0.09 \mathrm{a}$ & $1.06 \pm 0.16 \mathrm{~b}$ \\
\hline \multicolumn{5}{|c|}{ Massive blooming } \\
\hline \multirow{3}{*}{ Methanol } & $99.8 \%$ & $2.36 \pm 0.07 c$ & $2.19 \pm 0.09 \mathrm{~b}$ & $3.38 \pm 0.33 c$ \\
\hline & $70 \%$ & $1.79 \pm 0.04 \mathrm{c}$ & $1.73 \pm 0.17 \mathrm{c}$ & $4.10 \pm 0.14 \mathrm{~b}$ \\
\hline & $40 \%$ & $1.51 \pm 0.28 b$ & $2.45 \pm 0.16 c$ & $3.94 \pm 0.13 c$ \\
\hline \multirow{3}{*}{ Ethanol } & $96 \%$ & $0.72 \pm 0.05 c$ & $1.11 \pm 0.13 b$ & $2.84 \pm 0.30 \mathrm{c}$ \\
\hline & $70 \%$ & $0.86 \pm 0.10 c$ & $3.05 \pm 0.36 c$ & $2.87 \pm 0.30 \mathrm{c}$ \\
\hline & $40 \%$ & $1.79 \pm 0.05 c$ & $2.65 \pm 0.08 \mathrm{c}$ & $2.94 \pm 0.06 \mathrm{c}$ \\
\hline \multirow{3}{*}{ Isopropanol } & $99.5 \%$ & $0.22 \pm 0.07 c$ & $0.25 \pm 0.02 b$ & $1.24 \pm 0.27 \mathrm{c}$ \\
\hline & $70 \%$ & $2.13 \pm 0.38 \mathrm{c}$ & $2.47 \pm 0.19 c$ & $3.23 \pm 0.19 c$ \\
\hline & $40 \%$ & $1.74 \pm 0.05 c$ & $1.83 \pm 0.03 c$ & $3.44 \pm 0.19 c$ \\
\hline \multicolumn{2}{|l|}{ Water } & n.a. & $0.72 \pm 0.09 \mathrm{a}$ & $0.46 \pm 0.05 c$ \\
\hline \multicolumn{5}{|c|}{ Fruit ripening } \\
\hline \multirow{3}{*}{ Methanol } & $99.8 \%$ & $7.72 \pm 0.04 \mathrm{a}$ & $8.68 \pm 0.30 \mathrm{a}$ & $8.35 \pm 0.04 a$ \\
\hline & $70 \%$ & $9.28 \pm 0.23 \mathrm{a}$ & $9.96 \pm 0.21 \mathrm{a}$ & $8.34 \pm 0.32 \mathrm{a}$ \\
\hline & $40 \%$ & $7.18 \pm 0.38 \mathrm{a}$ & $9.53 \pm 0.12 \mathrm{a}$ & $10.77 \pm 0.24 \mathrm{a}$ \\
\hline \multirow{3}{*}{ Ethanol } & $96 \%$ & $3.30 \pm 0.32 b$ & $5.74 \pm 0.27 \mathrm{a}$ & $8.94 \pm 0.21 \mathrm{a}$ \\
\hline & $70 \%$ & $3.32 \pm 0.14 b$ & $11.50 \pm 0.12 \mathrm{a}$ & $10.61 \pm 0.27 \mathrm{a}$ \\
\hline & $40 \%$ & $8.49 \pm 0.29 a$ & $10.59 \pm 0.14 \mathrm{a}$ & $10.13 \pm 0.19 a$ \\
\hline \multirow{3}{*}{ Isopropanol } & $99.5 \%$ & $9.28 \pm 0.25 \mathrm{a}$ & $3.85 \pm 0.35 \mathrm{a}$ & $5.47 \pm 0.05 \mathrm{a}$ \\
\hline & $70 \%$ & $10.72 \pm 0.35 a$ & $10.51 \pm 0.14 \mathrm{a}$ & $8.02 \pm 0.10 \mathrm{~b}$ \\
\hline & $40 \%$ & $8.76 \pm 0.32 \mathrm{a}$ & $9.73 \pm 0.22 \mathrm{a}$ & $8.01 \pm 0.31 b$ \\
\hline \multicolumn{2}{|l|}{ Water } & n.a. & n.a. & $1.76 \pm 0.05 \mathrm{a}$ \\
\hline
\end{tabular}

n.a. - no activity. The values marked with different letters differ significantly between development stages $(p<0.05$, $\mathrm{n}=3$ ) 
Table 5

Mean ( \pm standard deviation) total flavonoids content in fresh E. angustifolium herb extracts (in mg RU/g of raw material.

\begin{tabular}{|c|c|c|c|c|}
\hline \multicolumn{5}{|c|}{$\begin{array}{c}\text { Flavonoids } \\
\text { [mg rutin }(\mathrm{RU}) / \mathrm{g} \text { raw material] }\end{array}$} \\
\hline \multirow{2}{*}{ Solvent } & \multirow{2}{*}{$\begin{array}{l}\text { Alcohol concentration } \\
\qquad[v / v]\end{array}$} & \multicolumn{3}{|c|}{ Extraction time } \\
\hline & & $15^{\prime}$ & $30^{\prime}$ & $60^{\prime}$ \\
\hline \multicolumn{5}{|c|}{ Intensive growing } \\
\hline \multirow{3}{*}{ Methanol } & $99.8 \%$ & $4.89 \pm 0.36 \mathrm{ab}$ & $5.70 \pm 0.43 \mathrm{~b}$ & $5.26 \pm 0.16 \mathrm{~b}$ \\
\hline & $70 \%$ & $2.98 \pm 0.12 c$ & $4.43 \pm 0.10 \mathrm{a}$ & $4.76 \pm 0.06 \mathrm{a}$ \\
\hline & $40 \%$ & $1.14 \pm 0.08 \mathrm{c}$ & $3.58 \pm 0.12 b$ & $3.23 \pm 0.05 c$ \\
\hline \multirow{3}{*}{ Ethanol } & $96 \%$ & $4.06 \pm 0.09 \mathrm{a}$ & $4.90 \pm 0.32 \mathrm{a}$ & $3.20 \pm 0.10 \mathrm{~b}$ \\
\hline & $70 \%$ & $5.31 \pm 0.39 \mathrm{a}$ & $5.89 \pm 0.42 \mathrm{a}$ & $3.39 \pm 0.10 \mathrm{~b}$ \\
\hline & $40 \%$ & $3.28 \pm 0.10 \mathrm{a}$ & $4.48 \pm 0.13 \mathrm{a}$ & $2.90 \pm 0.11 \mathrm{~b}$ \\
\hline \multirow{3}{*}{ Isopropanol } & $99.5 \%$ & $2.20 \pm 0.07 b$ & $2.11 \pm 0.18 \mathrm{a}$ & $2.60 \pm 0.14 \mathrm{~b}$ \\
\hline & $70 \%$ & $3.49 \pm 0.09 b$ & $4.18 \pm 0.13 b$ & $6.26 \pm 0.07 a$ \\
\hline & $40 \%$ & $2.88 \pm 0.04 \mathrm{~b}$ & $2.80 \pm 0.15 b$ & $4.99 \pm 0.24 \mathrm{a}$ \\
\hline \multicolumn{2}{|l|}{ Water } & $0.72 \pm 0.02 \mathrm{a}$ & $1.38 \pm 0.10 \mathrm{a}$ & $0.92 \pm 0.05 \mathrm{a}$ \\
\hline \multicolumn{5}{|c|}{ Massive blooming } \\
\hline \multirow{3}{*}{ Methanol } & $99.8 \%$ & $4.42 \pm 0.31 \mathrm{~b}$ & $4.48 \pm 0.04 \mathrm{c}$ & $5.97 \pm 0.35 \mathrm{ab}$ \\
\hline & $70 \%$ & $3.65 \pm 0.15 b$ & $2.33 \pm 0.16 \mathrm{c}$ & $4.94 \pm 0.31 \mathrm{a}$ \\
\hline & $40 \%$ & $3.22 \pm 0.12 \mathrm{a}$ & $3.34 \pm 0.16 \mathrm{~b}$ & $3.62 \pm 0.02 b$ \\
\hline \multirow{3}{*}{ Ethanol } & $96 \%$ & $2.36 \pm 0.10 \mathrm{~b}$ & $2.30 \pm 0.15 \mathrm{c}$ & $3.44 \pm 0.21 \mathrm{~b}$ \\
\hline & $70 \%$ & $2.24 \pm 0.19 b$ & $4.14 \pm 0.17 b$ & $3.84 \pm 0.27 b$ \\
\hline & $40 \%$ & $2.91 \pm 0.07 b$ & $3.00 \pm 0.09 \mathrm{~b}$ & $3.43 \pm 0.23 b$ \\
\hline \multirow{3}{*}{ Iisopropanol } & $99.5 \%$ & $0.94 \pm 0.05 c$ & $0.85 \pm 0.12 b$ & $1.80 \pm 0.08 \mathrm{c}$ \\
\hline & $70 \%$ & $4.41 \pm 0.05 \mathrm{a}$ & $4.73 \pm 0.39 b$ & $5.06 \pm 0.07 \mathrm{c}$ \\
\hline & $40 \%$ & $2.80 \pm \pm 0.05 \mathrm{~b}$ & $2.82 \pm 0.20 \mathrm{~b}$ & $4.96 \pm 0.07 \mathrm{a}$ \\
\hline \multicolumn{2}{|l|}{ Water } & $0.81 \pm 0.03 \mathrm{a}$ & $0.70 \pm 0.12 b$ & $1.00 \pm 0.04 \mathrm{a}$ \\
\hline \multicolumn{5}{|c|}{ Fruit ripening } \\
\hline \multirow{3}{*}{ Methanol } & $99.8 \%$ & $5.38 \pm 0.20 \mathrm{a}$ & $7.03 \pm 0.20 \mathrm{a}$ & $6,21 \pm 0,51 \mathrm{a}$ \\
\hline & $70 \%$ & $4.06 \pm 0.12 \mathrm{a}$ & $3.76 \pm 0.41 \mathrm{~b}$ & $4,94 \pm 0,56 a$ \\
\hline & $40 \%$ & $2.78 \pm 0.14 b$ & $4.22 \pm 0.10 \mathrm{a}$ & $4,68 \pm 0,19 a$ \\
\hline \multirow{3}{*}{ Ethanol } & $96 \%$ & $2.32 \pm 0.08 \mathrm{~b}$ & $4.20 \pm 0.20 \mathrm{~b}$ & $5,18 \pm 0,41 \mathrm{a}$ \\
\hline & $70 \%$ & $1.87 \pm 0.17 \mathrm{~b}$ & $5.25 \pm 0.21 \mathrm{a}$ & $4,48 \pm 0,18 \mathrm{a}$ \\
\hline & $40 \%$ & $2.71 \pm 0.08 b$ & $4.72 \pm 0.12 \mathrm{a}$ & $4,27 \pm 0,46 a$ \\
\hline \multirow{3}{*}{ Isopropanol } & $99.5 \%$ & $3.00 \pm 0.12 \mathrm{a}$ & $2.43 \pm 0.11 \mathrm{a}$ & $3,67 \pm 0,20 \mathrm{a}$ \\
\hline & $70 \%$ & $3.85 \pm 0.30 \mathrm{~b}$ & $5.40 \pm 0.18 \mathrm{a}$ & $5,46 \pm 0,07 b$ \\
\hline & $40 \%$ & $3.35 \pm 0.16 \mathrm{a}$ & $3.71 \pm 0.27 \mathrm{a}$ & $5,10 \pm 0,08 \mathrm{a}$ \\
\hline \multicolumn{2}{|l|}{ Water } & $0,95 \pm 0,15 \mathrm{a}$ & $0.85 \pm 0.09 \mathrm{~b}$ & $0.98 \pm 0.02 \mathrm{a}$ \\
\hline
\end{tabular}

The values marked with different letters differ significantly between development stages $(p<0.05, \mathrm{n}=3)$ 
stage after extraction with undiluted methanol during $30 \mathrm{~min}$ (tab. 5). Similar results were obtained by Deng et al. In their study the total polyphenol content in ethanol extracts from E. angustifolium, was $16.8 \mathrm{~g}$ GA/100 g extract [22]. Also Wojdyło et al. determined the total polyphenol of $4.03 \mathrm{mg} \mathrm{GA} / 100 \mathrm{~g}$ dry matter in extracts in $80 \%$ methanol [21].
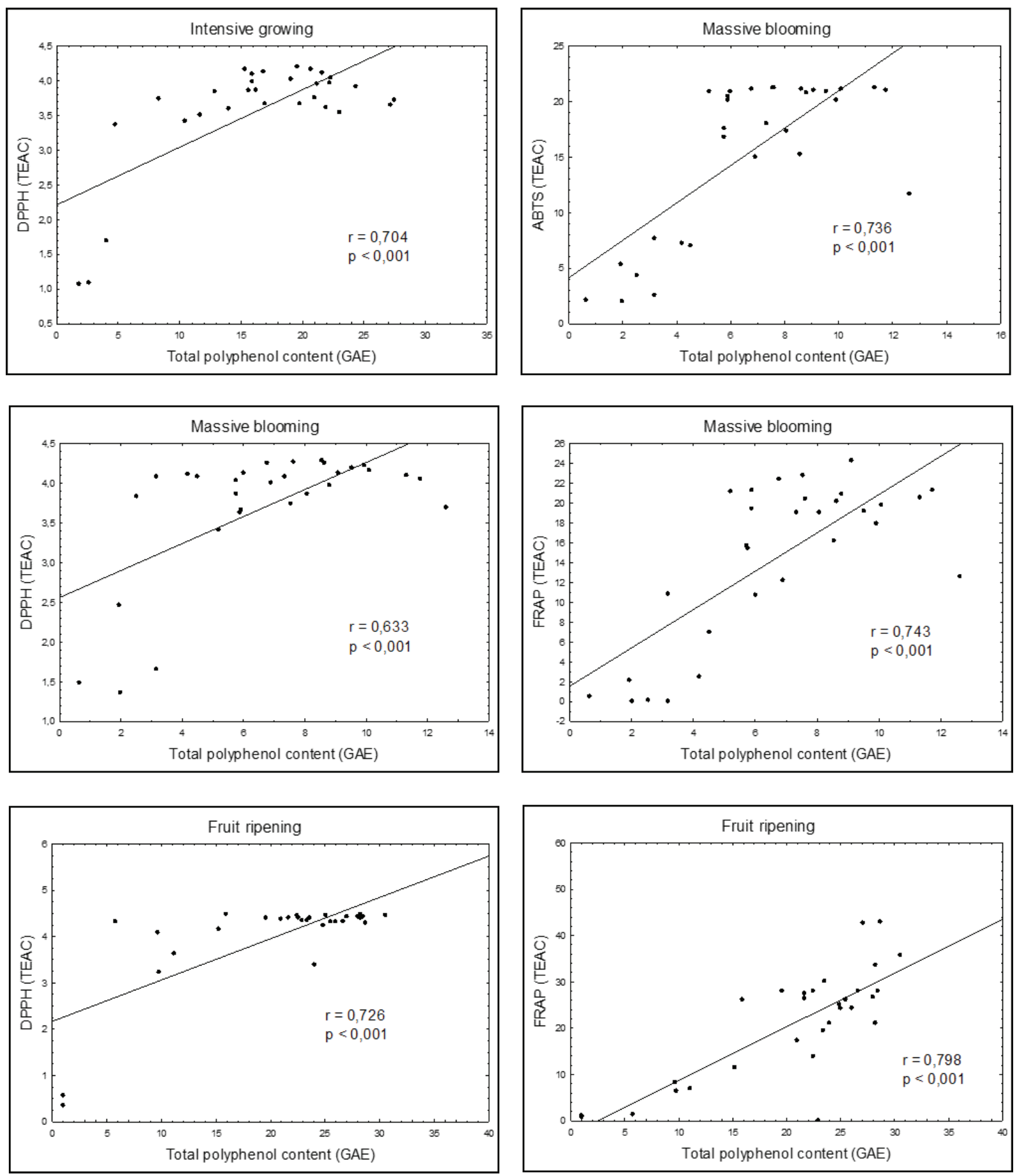

Figure 1

Correlations between antioxidant activities of fireweed extracts evaluated with different methods and total polyphenol content at different developmental stage.

TEAC - trolox equivalent antioxidant capacity (mg trolox/g raw material), GAE - gallic acid equivalent (mg gallic $\mathrm{acid} / \mathrm{g}$ raw material) 
highest statistically significant relationships between these parameters have been demonstrated for the massive blooming stage (fig. 1). A similar relationship was also found by Kaškoniene et al. They observed a significant linear relationship between antioxidant activity evaluated with DPPH and the total polyphenol content in E. angustifolium, the correlation coefficient was $r=0.98$ [24]. Wojdyło et al. observed a relationship between the total polyphenols content and antioxidant activity in plants of Labiaceae and Compositeae. In the first family included, among others, sage (Salvia officinalis), rosemary (Rosmarinus officinalis), common balm (Melissa officinalis), the correlation coefficient for these plants varied from 0.83 to 0.93 . However, in the case of Compositae family, included, among others, the elecampane (Inula helenium), dandelion (Taraxacum officinalis) or tansy (Tanacetum vulgare), the correlation coefficient was $r=0.67-0.96$ [21]. The above results suggest that the antioxidant effect of commonly used herbs depends to a high extent on their chemical composition. Other factors such as climatic and soil conditions as well as the plant's developmental stage can also influence plant biological activity [30]. The ability to scavenge free radicals may fluctuate depended on the vegetation phases. Maruška et al. demonstrated the highest antioxidant activity of E. angustifolium during the massive blooming phase [5]. In our study, in most cases, significant differences between particular vegetation phases were confirmed by Wilcoxon test. The differences between all phases of vegetation were demonstrated for DPPH, FRAP and Folin-Ciocalteu methods $(p=0.001)$. The highest values were observed after the comparison of DPPH, ABTS and the total polyphenols and flavonoids content occurred in the fruit ripening stage, whereas for FRAP technique in the intensive growing stage. This observation was confirmed by Kujawski et al. who found the highest concentration of E. angustifolium tannins in fruit ripening stage [2]. Maruška et al. observed the highest flavonoids content as well as antioxidant activity in massive blooming stage of E. angustifolium [5]. The observed differences between the studies might be partly due to other plant growth positions, associated with different climatic conditions. Diverse habitat conditions could have a significant impact on the accumulation of active substances in plants [24]. In our study, plants were harvested in Poland, whereas Maruška et al. evaluated plants from Lithuania. Jüngerson et al. analyzed the total polyphenols content in Estonia's individual parts of E. angustifolia, harvested from May to October, and found the highest amount of these substances in plants harvested in July [30].

Moreover, the solvent used for extraction may also influence the isolation of active substances [14, $32,33]$. Its polarity plays a key role in the determination of antioxidant activity and could significantly affect the transfer mechanisms of a single electron or hydrogen atom [34]. In our study, four solvents were used: ethanol, methanol, isopropanol and water. The highest antioxidant capacities were found for extracts in $70 \%$ and $96 \%$ ethanol, whereas the lowest in water extracts (tab. 1,2). In our previous study on the effect of various solvents and extraction time on antioxidant activity of green tea leaves extracts, it was found that in the case of the DPPH method undiluted methanol seemed to be the most preferred solvent for extracts obtained using ultrasound for 15 minutes, whereas for ABTS - 70\% methanol, in 60 minutes. We suggested that the best solvent for extraction of, for instance, polyphenols was water after $60 \mathrm{~min}$ extraction [14]. In present study, extracts of plant harvested in ripening fruit stage prepared in water during $60 \mathrm{~min}$ showed relatively higher values as compared to other aqueous extracts (tab. 2).

\section{CONCLUSION}

E. angustifolium is characterized by high antioxidant potential and high total polyphenols and flavonoids content. An important factor determining the accumulation of biologically active ingredients and, hence, the ability to scavenge free radicals, is the time of plant harvesting. The studied plant harvested in the second half of August and extracted with more concentrated ethanol are characterized by high antioxidant activity. Accordingly, E. angustifolium, a potential weed, could be consider as a beneficial source of antioxidants.

Conflict of interest: Authors declare no conflict of interest.

\section{REFERENCES}

1. Granica S, Piwowarski JP, Czerwińska ME, Kiss AK. Phytochemistry, pharmacology and traditional uses of different Epilobium species (Onagraceae): a review. J Ethnopharmacol 2014; 156:316-46. doi: http://dx.doi.org/10.1016/j. jep.2014.08.036

2. Kujawski R, Bogacz A, Derebecka-Hołysz N, Cichocka J, Kujawski J, Mikołajczak P£, et al. 
Rośliny lecznicze z rodzaju Epilobium - działanie biologiczne i farmakologiczne [Medicinal plants from Epilobium genus - biological and pharmacological properties]. Herba Pol 2010; 56(1):6782 [in Polish].

3. Kiss AK, Bazylko A, Filipek A, Granica S, Jaszewska E, Kiarszys U, et al. Oenothein B's contribution to the anti-inflammatory and antioxidant activity of Epilobium sp. Phytomedicine 2011; 18:557-60. doi: http://dx.doi.org/10.1016/j. phymed.2010.10.016

4. Pawicka K, Woziwoda B, Wolski GJ. Flora roślin naczyniowych rezerwatu "Jamno" [Diversity of vascular plant species in "Jamno" nature reserve]. Biuletyn Szadkowski 2012; 12:145-57 [in Polish].

5. Maruška A, Ragažinskienė $O$, Vyšniauskas $O$, Kaškonienė V, Bartkuviené V, Kornyšova $O$, et al. Flavonoids of willow herb (Chamerion angustifolium (L.) Holub) and their radical scavenging activity during vegetation. Adv Med Sci. 2014; 59(1):136-41. doi: http://dx.doi.org/10.1016/j.advms.2013.10.002

6. European Union herbal monograph on Epilobium angustifolium L. and/or Epilobium parviflorum Schreb., herba. European Medicines Agency, EMA/HMPC/712511/2014.

7. Jang IC, Jo EK, Bae MS, Lee HJ, Jeon GI, Park E, et al. Antioxidant and antigenotoxic activities of different parts of persimmon (Diospyros kaki cv. Fuyu) fruit. J Med Plant Res 2010; 4(2):155-60.

8. Ścibior-Bentkowska D, Czeczot H. Komórki nowotworowe a stres oksydacyjny. [Cancer cells and oxidative stress]. Postępy Hig Med Dośw 2009; 63:58-72.

9. Zabłocka A, Janusz M. Dwa oblicza wolnych rodników tlenowych. [Two faces of reactive oxygen species]. Postępy Hig Med Dośw 2008; 62:118-24.

10. Chemat F, Vian MA, Cravotto G. Green extraction of natural products: concept and principles. Int J Mol Sci 2012; 13:8615-27. doi: http://dx.doi. org/10.3390/ijms13078615

11. Wang J, Sun B, Cao Y, Tian Y, Li X. Optimisation of ultrasound-assisted extraction of phenolic compounds from wheat brain. Food Chem 2008; 106:804-10. doi: http://dx.doi.org/10.1016/j. foodchem.2007.06.062

12. Nowak A, Zielonka-Brzezicka J, Pechaiko D, Tkacz M, Klimowicz A. Ocena właściwości antyoksydacyjnych liści Ginkgo biloba L. po zakończeniu wegetacji. [The evaluation of the antioxidant properties of Ginkgo biloba L. leaves after the end of the growing season]. Pomeranian J Life Sci 2017; 63(1):9-15. doi: http://dx.doi. org/10.21164/pomjlifesci.222

13. Zielonka-Brzezicka J, Nowak A, Klimowicz A, Wira D, Grzesiak K, Rędzikowska E, et al. Aktinidia chińska jako źródło prozdrowotnych antyoksydantów [Actinidia chinensis as a source of health-promoting antioxidants]. Probl Hig Epidemiol 2018; 99(3):238-244.

14. Nowak A, Maciejewska A, Duchnik W, Florkowska K, Klimowicz A. Wpływ rozpuszczalnika na właściwości antyoksydacyjne ekstraktów z zielonej herbaty (Camellia sinensis L.) [The effect of a solvent on the antioxidant properties of green tea (Camellia sinensis L.) extracts]. Probl Hig Epidemiol 2018; 99(3):245-58 [in Polish].

15. Florkowska K, Duchnik W, Nowak A, Klimowicz A. Właściwości antyoksydacyjne papryki ostrej odmiany Hungarian yellow [The antioxidant activity of Hungarian yellow hot pepper]. Pomeranian J Life Sci 2018; 64(3):126-31 [in Polish]. doi: http://dx.doi.org/10.21164/pomjlifesci.455

16. Barreira JCM, Ferreira ICFR, Oliveira MBPP, Pereira JA. Antioxidant activities of the extracts from chestnut flower, leaf, skins and fruit. Food Chem 2008; 107(3):1106-13. doi: http://dx.doi. org/10.1016/j.foodchem.2007.09.030

17. Li Y, Fabiano-Tixier AS, Tomao V, Cravotto G, Chemat F. Green ultrasound-assisted extraction of carotenoids based on the bio-refinery concept using sunflower oil as an alternative solvent. Ultrason Sonochem 2013; 20:12-8. doi: http:// dx.doi.org/10.1016/j.ultsonch.2012.07.005

18. Goula AM, Ververi M, Adamopoulou A, Kaderides K. Green ultrasound-assisted extraction of carotenoids from pomegranate wastes using vegetable oils. Ultrasound Sonochem 2017; 34:821-830. doi: http://dx.doi.org/10.1016/j.ultsonch.2016.07.022

19. Chemat F, Rombaut N, Sicaire AG, Meullemies- 
tre A, Fabiano-Tixier AS, Abert-Vian M. Ultrasound-assisted extraction of food and natural products. Mechanism, techniques, combinations, protocols and applications. A review. Ultrason Sonochem 2017; 34:540-60. doi: http://dx.doi. org/10.1016/j.ultsonch.2016.06.035

20. Zych I, Krzepiłko A. Pomiar całkowitej zdolności antyoksydacyjnej wybranych antyoksydantów i naparów metodą redukcji rodnika DPPH [Measurement of total antioxidant capacity of selected antioxidants and infusions using DPPH radical reduction]. Chem Dydakt Ekol Metrol 2010; 15(1):51-4 [in Polish].

21. Wojdyło A. Oszmiański J, Czemerys R. Antioxidant activity and phenolic compounds in 32 selected herbs. Food Chem 2007; 105:940-9. doi: http:// dx.doi.org/10.1016/j.foodchem.2007.04.038

22. Deng LQ, Zhou SY, Mao JX, Liu S, Lan XZ, Liao $\mathrm{ZH}$, et al. HPLC-ESI-MS/MS analysis of phenolics and in vitro antioxidant activity of Epilobium angustifolium L. Nat Prod Res 2018; 32(12):14325. doi: http://dx.doi.org/10.1080/14786419.2017. 1344659

23. Schepetkin IA, Ramstead AG, Kirpotina LN, Voyich JM, Jutila MA, Quinn MT. Therapeutic potential of polyphenols from Epilobium angustifolium (Fireweed). Phytother Res 2016; 30:128797. doi: http://dx.doi.org/10.1002/ptr.5648.

24. Kaškonienė V, Maruška A, Akuñeca I, Stankevičius M, Ragažinskienè O, Bartkuvienè V, et al. Screening of antioxidant activity and volatile compounds composition of Chamerion angustifolium (L.) Holub ecotypes grown in Lithuania. Nat Prod Res 2016; 30(12):1373-1381. doi: http:// dx.doi.org/10.1080/14786419.2015.1058792

25. Tóth BH, Blazics B, Kéry Á. Polyphenol composition and antioxidant capacity of Epilobium species. J Pharm Biomed Anal 2009; 49:26-31. doi: http://dx.doi.org/10.1016/j.jpba.2008.09.047

26. Ştef DS, Gergen I, Traşcă TI, Hărmănescu M, Lavinia Ş, Ramona B, et al. Total antioxidant and radical scavenging capacities for different medicinal herbs. Rom Biotech Lett 2009; 14(5):4705-10. doi: http:// dx.doi.org/10.1080/14786419.2015.1058792
27. Cybul M, Nowak R. Przegląd metod stosowanych $\mathrm{w}$ analizie właściwości antyoksydacyjnych wyciągów roślinnych [Review of the methods applied to measuring of antioxidant activity of plant extracts]. Herba Pol 2008; 54(1):68-78 [in Polish].

28. Zalega J, Szostak-Węgierek D. Żywienie w profilaktyce nowotworów. Część I. Polifenole roślinne, karotenoidy, błonnik pokarmowy [Nutrition in cancer prevention. Part I. Plant polyphenols, carotenoids, dietary fiber]. Probl Hig Epidemiol 2013; 94(1):41-9 [in Polish].

29. Vitalone A, Allkanjari O. Epilobium spp: pharmacology and phytochemistry. Phytother Res 2018; 32:1229-40. doi: http://dx.doi.org/10.1002/ ptr.6072

30. Łata B, Wińska-Krysiak M, Lewandowska J. Potencjał przeciwutleniający owoców borówki wysokiej w zależności od odmiany i roku badań [The antioxidant potential of blueberry fruits: year and cultivar variation]. Rocz AR w Poz 2007; 383(41):339-344 [in Polish].

31. Jürgenson S, Matto V, Raal A. Vegetational variation of phenolic compounds in Epilobium angustifolium. Nat Prod Res 2012; 26(20):1951-3. doi: http://dx.doi.org/10.1080/14786419.2011.643310

32. Wianowska D, Wiśniewski M. Simplified procedure of silymarin extraction from Silybum marianum L. Gaertner. J Chrom Sci 2015; 53(2):366-72. doi: http://dx.doi.org/10.1093/chromsci/bmu049

33. Gawlik-Dziki U, Kowalczyk D. Wpływ warunków ekstrakcji na aktywność przeciwutleniającą ekstraktów z kiełków rzodkiewki [Influence of extraction conditions on antioxidant activity of extracts from radish sprouts]. Żywn Nauk Technol Ja 2007; 1(50):132-9 [in Polish].

34. Pawlak S, Sielicka M. Wpływ rozpuszczalnika zastosowanego do ekstrakcji na oznaczoną zawartość związków fenolowych i aktywność przeciwutleniającą wytłoków $\mathrm{z}$ aronii. In: Górecka D, Pospiecha E. Zagospodarowanie ubocznych produktów przemysłu spożywczego. Poznań: Wydział Nauk o Żywności i Żywieniu, Uniwersytet Przyrodniczy w Poznaniu 2016; 2535 [in Polish]. 